\title{
Epinephrine-cue contiguity is required for the modulation of CS excitatory strength
}

\author{
M. J. KELLEY \\ University of London, London, England
}

\begin{abstract}
The present study shows that contiguity of elevated epinephrine levels with the presence of a fear cue is a necessary condition to produce permanent associative changes in the excitatory strength of the cue. Rats were first given three .5-mA footshocks during two direct placements in the black side of a shuttlebox with a closed guillotine door; they were never shocked during two placements on the white side. In the second phase, the rats were reexposed to the black side and given a prior $.02 \mathrm{mg}$ injection of epinephrine, a prior .02-mg injection of saline, or an epinephrine injection $5 \mathrm{~h}$ later. The latency to cross from the white to black side $24 \mathrm{~h}$ later was found to be considerably longer in the groups given an epinephrine injection shortly before reexposure.
\end{abstract}

After Pavlovian conditioning, a conditioned stimulus (CS) presented alone may in some cases fail to show a decrement in excitatory strength, and may instead even show a permanent increment. Although Eysenck (1979) has called such occurrences "incubation effects," other theorists have used the term "reminder effect"' (Riccio \& Concannon, 1981). Haroutunian and Riccio (1977) provided some evidence that differences in levels of epinephrine may be an important factor in incubation effects. In their study a group of rats that received an injection of epinephrine followed by a reexposure to a fear CS showed a several-fold increase in the excitatory properties of the cue, as measured by approach latencies the following day when the rats were in a nondrug state. This did not occur in control groups receiving no exposure, exposure to the CS alone, or the epinephrine injection without exposure. Although an associative mechanism is implicated, the controls the authors provide are insufficient to allow the inference that associative contiguity between increased levels of epinephrine and CS-only exposure is necessary to obtain the incubation effect. To make that inference requires the use of a control group that receives unpaired exposure to both the hormone and the CS. The present report provides a partial replication of the Haroutunian and Riccio (1977) study, and demonstrates that it is the actual contiguous exposure to the CS with elevations of epinephrine that produces this permanent change in the excitatory properties of a CS.

\section{METHOD}

\section{Subjects}

The subjects were experimentally naive Wister-derived rats from the breeding colony maintained at the Psychology Laboratory at

Support for this study was supplied by a special grant to H. J. Eysenck from the Tobacco Research Council, USA. The author's mailing address is Department of Psychology, University of Hawaii, Honolulu, HI 96822.
Bethlehem Royal Hospital. They were housed in groups of 3 under ad-lib feeding conditions.

\section{Apparatus}

The apparatus was a commercial shuttlebox with a guillotine door between the two $20 \times 24 \times 20 \mathrm{~cm}$ compartments. The inside surface of one compartment was flat white and the other was flat black. The grid bars on the white side of the box were covered with a white piece of wood. Lighting was supplied by a 24-v bulb with a clear lens cap at each end of the shuttlebox. Placement of the shuttlebox in a sound-attenuated chamber and 75-dB white noise shielded the animals from extraneous sounds. A wire connected to the guillotine door and passing through the sound-attenuated chamber allowed the door to be raised $10 \mathrm{~cm}$. Constant-current shock to the grid bar in the black compartment was supplied by a Cambridge Bioscience shock scrambler. A 250-g weight centered on the grid floor was sufficient to depress the floor slightly and thus allow for the electromechanical timing of the latencies to cross on test trials.

\section{Procedures}

Two three-stage experiments were performed. In the first experiment, 20 120-day-old rats were handled six times for 2-3 min and were randomly assigned to either a saline or an epinephrine group. On the training day, individual rats (in a counterbalanced order) were twice given two direct 3-min exposures to each side of the shuttlebox. The initial placement was always on the white side and the three subsequent placements were alternated between sides. The connecting door was always closed. During the two exposures to the black side, a .5-sec .5-mA shock was given at 60 -sec intervals (three shocks per exposure). The following day all of the rats were reexposed to the black side for $60 \mathrm{sec}$ but no shocks were given. Twenty minutes prior to this reexposure, the rats were briefly taken out of their cages and given a .5 -ml subcutaneous injection of $.02 \mathrm{mg}$ of either epinephrine or saline. The epinephrine (Sigma E4250) was mixed immediately beforehand by first dissolving it in $\mathrm{HCl}$ and then diluting it in saline. Oxidation was slowed by adding a pinch of ascorbic acid. Equivalent amounts of $\mathrm{HCl}$ and ascorbic acid were added to the saline solution for the control group. Twenty-four hours later the rats were tested for their latency to cross from the white to black side. These test trials began with the rats' being placed on the white side and the raising of the guillotine door $20 \mathrm{sec}$ later.

The second experiment used the same apparatus and general procedures of the first experiment, except that the 16 rats were younger ( 80 days old) and were handled only four times for $3 \mathrm{~min}$. In this 
experiment, both groups received the epinephrine injection and were reexposed to the CS $24 \mathrm{~h}$ after training, but the first group received its injection $20 \mathrm{~min}$ prior to reexposure to the cue and the second group received the injection $5 \mathrm{~h}$ after the reexposure session. The crossover latencies were tested $24 \mathrm{~h}$ after the second group was injected.

\section{RESULTS AND DISCUSSION}

The results suggest that a permanent increase in the excitatory properties of a fear cue can be produced by contiguous exposure to high levels of epinephrine and the CS. In the first experiment, the mean latency to cross from the white to black side was $514 \mathrm{sec}$ for the group given an injection of epinephrine prior to reexposure, but in the group given a saline injection the mean latency was $150 \mathrm{sec}$. This difference is reliable $[\mathrm{t}(19)=2.99, \mathrm{p}<$ $.05]$. In the second experiment, the group given epinephrine prior to reexposure had a mean latency of $567 \mathrm{sec}$ to crossover on the test trial, and the group given the injection $5 \mathrm{~h}$ after reexposure had a mean latency of $59 \mathrm{sec}$. The difference between these means is also reliable [ $t(19)$ $=3.60, \mathrm{p}<.01]$. The group receiving the injection several hours after the reexposure had a nearly 10 -fold faster latency on the final test trial (i.e., less fear) compared to the group which had the injection shortly before the reexposure, thus demonstrating that associative contiguity of high levels of epinephrine with exposure to the $\mathrm{CS}$ is required to obtain this incubation effect.

Using a design similar to that used in their 1977 study, Haroutunian and Riccio (1979) demonstrated that ACTH would also induce incubation effects. More recently, Richardson, Riccio, and Devine (1984) showed that an injection of ACTH (in the form of Acthar Gel; Armour) some $24 \mathrm{~h}$ prior to reexposure to the cue was sufficient to produce an incubation effect. As saline rather than gel without the ACTH was used in the control groups, Richardson et al.'s findings are most likely attributable to properties of the gel, or to the gel's prolonging the halflife of the ACTH. The results of the present study support the earlier findings of Rigter, Elbertse, and van Reizen (1975) that CS contiguity with hormone levels is necessary.

It is unclear what psychological mechanisms are involved in these instances of hormone-mediated incubation effects. Haroutunian and Riccio (1979) argue that it is the representation of the endogenous physiological cues present during original training contiguous with the CS that results in further learning. This explanation requires, as the authors point out, an assumption of state-dependent learning; however, this form of learning seldom occurs when salient environmental cues (such as were present in their study) reliably predict the reinforcer (Eich, 1980). A second possibility is that increased ACTH levels (whether induced by injections of epinephrine, ACTH, or a corticotropin-releasing factor) may be anxiogenic (File \& Velluci, 1978; Sutton, Koob, Le Moal, River, $\&$ Vale, 1982). Other authors have argued for the involvement of attention mechanisms and have said that a fear hypothesis is insufficient (Beckwith \& Sandman, 1982). It is also not certain that epinephrine is producing these incubation effects by interacting with corticotropinreleasing factor in the release of ACTH (Borrell, De Kloet, Versteeg, \& Bohus, 1983; McGaugh, 1983; Axelrod \& Reisine, 1984). It is clear only that these effects induced by elevations in levels of hormones are reliable and dependent upon contiguity of the CS with neuroendocrine changes. Given the robustness of these instances of hormone-induced incubation, there may be justification for considering this as a model of clinical anxiety in humans (Eysenck \& Kelley, in press).

\section{REFERENCES}

Axelrod, J., \& Reisine, T. D. (1984). Stress hormones: Their interaction and regulation. Science, 224, 452-459.

Beckwith, B. E., \& Sandman, C. A. (1982). Central nervous system and peripheral effects of ACTH, MSH, and related neuropeptides. Peptides, 3, 411-420.

Borrell, J., De Kloet, E. R., Versteeg, D. H. G., \& Bohus, B. (1983). Inhibitory avoidance deficit following short-term adrenalectomy in the rat: The role of adrenal catecholamines. Behavioral \& Neural Biology, 39, 241-258.

EICH, J. E. (1980). The cue-dependent nature of state-dependent retrieval. Memory \& Cognition, 8, 157-173.

EYSENCK, H. J. (1979). The conditioning model of neurosis. Behavior \& Brain Science, 2, 155-199.

EYSENCK, H. J., \& KELLEY, M. J. (in press). The interaction of neurohormones with Pavlovian A and Pavlovian B conditioning in the causation, extinction and incubation of anxiety. In G. Davey (Ed.), Conditioning in Humans. London: Wiley.

File, S. E., \& VellucCI, S. V. (1978). Studies on the role of ACTH and of 5-HT in anxiety, using an animal model. Journal of Pharmacy Pharmacology, 30, 105-110.

Haroutunian, V., \& Riccio, D. C. (1977). Effect of arousal conditions during reinstatement treatment upon learned fear in young rats. Developmental Psychobiology, 10, 25-32.

Haroutunian, V., \& Riccio, D. C. (1979). Drug-induced "arousal" and the effectiveness of CS exposure in the reinstatement of memory. Behavioral \& Neural Biology, 26, 115-120.

McGaugh, J. L. (1983). Hormonal influences on memory. Annual Review of Psychology, 34, 297-323.

Riccio, D. C., \& Concannon, J. T. (1981). ACTH and the reminder phenomena. In J. L. Martinez, Jr., R. A. Jensen, \& R. B. Messing (Eds.), Endogenous peptides and learning and memory processes (pp. 117-142). New York: Academic Press.

Richardson, R., Riccio, D. C., \& Devine, L. (1984). ACTH-induced recovery of extinguished avoidance responding. Physiological Psychology, 12, 184-192.

RigTer, H., ElberTSe, R., \& VAN Reizen, H. (1975). Time-dependent anti-amnesic effect of ACTH4-10 and desglycinamide-lysine vasopressin. Progress in Brain Research, 42, 163-171.

Sutton, R. E., Koob, G. F., Le Moal, M., River, J., \& Vale, W. (1982). Corticotropin-releasing factor produces behavioural activation in rats. Nature, 297, 331-333.

(Manuscript received March 30, 1985; revision accepted for publication August 23, 1985.) 